\title{
A comparative study of solution phase as well as solvent free microwave assisted syntheses of 3-benzothiazole/isoxazole substituted 2-styryl-4(3H)-quinazolinones
}

\author{
Chandresh L. Jagani, ${ }^{\text {a Satish F. Vanparia, }}{ }^{\text {a }}$ Tarosh S. Patel, ${ }^{\text {a }}$ \\ Ritu B. Dixit, ${ }^{b}$ Bharat C. Dixit*a \\ ${ }^{a}$ V. P. \& R. P. T. P. Science College, Affiliated to Sardar Patel University, \\ Vallabh Vidyanagar - 388120, Gujarat, India \\ ${ }^{b}$ Ashok \& Rita Patel Institute of Integrated Studies and Research in Biotechnology and Allied \\ Sciences, New Vallabh Vidyanagar - 388121, Gujarat, India \\ E-mail: dixits20002003@yahoo.co.in
}

\begin{abstract}
An optimization of the reaction conditions in solvent free and solution phase microwave assisted synthesis of 2-styryl-4(3H)-quinazolinone derivatives having substituted benzothiazole/5-tertbutylisoxazole at the $3^{\text {rd }}$ position is discussed. In solvent free reactions acidic alumina was used as a solid support, while a mixture of DMF and pyridine was used in solution phase synthesis. A comparative study of yield and reaction time for both the optimized method revealed that the solution phase microwave irradiation gave better results than solvent free method.
\end{abstract}

Keywords: Solvent free, solution phase, microwave, acidic alumina, quinazolinone

\section{Introduction}

Industrialization has led to immense environmental deterioration. The increasing environmental consciousness all over the world has put a pressing need to develop an alternative synthetic approach for synthetically and biologically important compounds. This requires a new approach, which will reduce the material and energy intensity of chemical processes and products, to minimize or eliminate the dispersion of harmful chemicals in the environment in a way that enhances industrially benign approach and meets the challenges of green chemistry. ${ }^{1}$

Microwave-assisted organic synthesis (MAOS) continues to affect synthetic chemistry significantly by enabling rapid, reproducible and scalable chemistry development. ${ }^{2}$ Numerous reactions including condensations, cycloadditions, heterocycle formations, and metal catalyzed cross-coupling have been explored under microwave conditions. ${ }^{3}$ MAOS can facilitate the discovery of new reactions and reduce cycle time in optimization of reactions. In addition, it 
serves to expand chemical space in compound library synthesis. ${ }^{4}$ Moreover, in recent years, application of microwave irradiation and recyclable, less expensive solid supports for organic transformation is emerging significantly. The microwave irradiation in conjugation with the use of solid supported reagents under solvent free condition provides a unique chemical process with special attributes such as enhanced reaction rates, higher yields of pure products, easier work-up and rapid optimization of reactions in parallel and several eco-friendly advantages in the context of green chemistry. ${ }^{5}$

The quinazolinone core and its derivatives form an important class of compounds, as they are found in a range of compounds exhibiting a broad spectrum of biological effects. ${ }^{6}$ Among the various classes of 4(3H)-quinazolinones, 2-styrylquinazolin-4(3H)-ones has attracted much attention as a tubulin polymerization inhibitor, ${ }^{7}$ cytotoxic agent, ${ }^{8}$ Hsp90 inhibitor, ${ }^{9}$ anticonvulsant, ${ }^{10}$ sedative-hypnotic, ${ }^{11}$ antibacterial and antifungal agent. ${ }^{12}$

In continuation of our previous reports ${ }^{13}$ and looking to the importance of the solid supported solvent free reactions and microwave technology, we decided to synthesize newer $(E)$-3benzothiazole/isoxazole substituted 2-styryl quinazolin-4(3H)-one derivatives (1) using solvent free solid support techniques under microwave irradiation. In order to compare the results of solvent free technique, the synthesis of the all the compounds (1a-1u) was repeated using solution phase microwave irradiation.

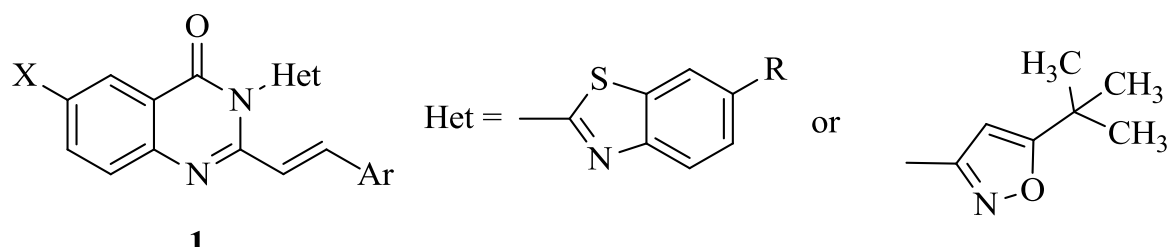

\section{Results and Discussion}

The majority of reported 4(3H)-quinazolinone derivatives were synthesized either from anthranilic acid or its derivatives. ${ }^{14}$ There are number of reports on one-pot synthesis of quinazoline-4(3H)-ones under solvent free conditions but, it involves the use of alkyl orthoesters which limits the variety of substitutions at $2^{\text {nd }}$ position. ${ }^{15}$ All attempts to prepare $(E)-3$ benzothiazole/isoxazole substituted-2-styryl quinazolin-4(3H)-one derivatives (1) directly from anthranilic acid or from $N$-acylanthranilic acids, under solvent free conditions were unsuccessful. Therefore, various (E)-2-styryl-4H-3,1-benzoxazin-4-ones (2) were prepared and used as a starting material. Initially cinnamoyl chloride was condensed with anthranilic acid in presence of pyridine at $0{ }^{\circ} \mathrm{C}$ to afford $N$-cinnamoyl anthranilic acids which was then cyclized to $(E)$-2-styryl4H-3,1-benzoxazin-4-one by refluxing it with acetic anhydride. However, a sufficient amount of (E)-2-styryl-4H-3,1-benzoxazin-4-one was obtained directly, when the condensation was carried out at room temperature. 
Recently, Zhou and Gregor et al. developed a general method for the synthesis of 3sulfonamide-substituted quinazolinone derivatives by condensation of benzoxazinones and substituted sulfonyl hydrazides under solvent free conditions at $130{ }^{\circ} \mathrm{C}$ (Scheme 1). ${ }^{17}$ However, when the mixture of $(E)$-2-styryl-4H-3,1-benzoxazin-4-one (2a, $\mathrm{X}=\mathrm{H}, \mathrm{Ar}=\mathrm{Ph})$ and 2-amino-1,3benzothiazole (3a) was heated under microwave irradiation, the reaction mixture did not give desired product by melting-fusion method. Therefore, we decided to use solid support strategy.

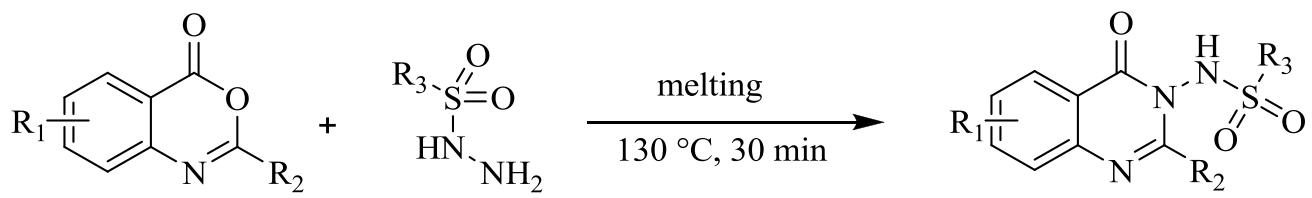

\section{Scheme 1}

For the preparation of quinazolinones from benzoxazinones, the use of silica gel under microwave irradiation has already been reported. ${ }^{18}$ However, the same protocol or dicyclohexyl carbodiimide $^{18}$ (DCC) with silica gel did not work when applied for the synthesis of compounds 1a-1u. After that various other supports viz. acidic alumina, ${ }^{19}$ silica sulfuric acid $^{20}$ had been attempted. Out of which, acidic alumina gave good results, while silica sulfuric acid resulted into the formation of complex reaction mixture.

Better results found in presence of acidic alumina encouraged us to further optimize the reaction conditions by varying the amount of alumina and power level of the microwave irradiation. The optimized reaction condition (Scheme 2) including $4 \mathrm{~g}$ of alumina per 2 mmole of benzoxazinone (2a, $\mathrm{X}=\mathrm{H}, \mathrm{Ar}=\mathrm{Ph})$ at $455 \mathrm{~W}$, yielded the required product $\mathbf{1 a}$ in $67 \%$ yield (Table 1, Entry 7).

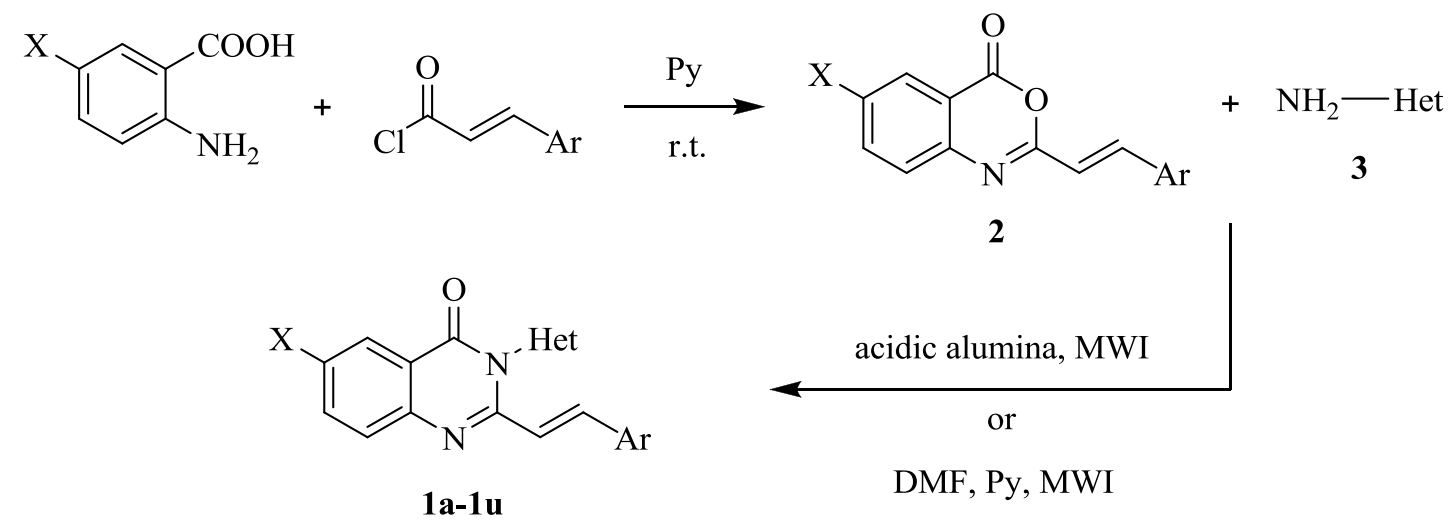

\section{Scheme 2}


Table 1. Optimization of reaction conditions for acidic alumina supported synthesis of (E)-3(1,3-benzothiazol-2-yl)-2-styrylquinazolin-4(3H)-one (1a) under microwave irradiation.

\begin{tabular}{ccccc}
\hline Entry & $\begin{array}{c}\text { Amount of alumina } \\
(\mathrm{gm})\end{array}$ & $\begin{array}{c}\text { Power } \\
(\mathrm{W})\end{array}$ & $\begin{array}{c}\text { Reaction time } \\
(\mathrm{min})\end{array}$ & $\begin{array}{c}\text { Yield }^{\mathrm{b}} \\
(\%)\end{array}$ \\
\hline 1 & 2 & 350 & 15 & 42 \\
2 & 3 & 350 & 14 & 55 \\
3 & 4 & 350 & 14 & 62 \\
4 & 5 & 350 & 11 & 57 \\
5 & 4 & 280 & 15 & 40 \\
6 & 4 & 420 & 9 & 64 \\
7 & 4 & 455 & 7 & 67 \\
8 & 4 & 490 & 6 & 65 \\
\hline
\end{tabular}

${ }^{\mathrm{a}}$ per 2 mmole of (E)-2-styryl-4H-3,1-benzoxazin-4-one (2a). ${ }^{\mathrm{b}}$ isolated yield after column chromatography.

For the sake of comparison, the same reaction was repeated in presence of DMF because of its excellent solvating property and high loss tangent. ${ }^{21}$ The required compound $(\mathbf{1 a}, \mathrm{X}=\mathrm{H}$, $\mathrm{Ar}=\mathrm{Ph}, \mathrm{Het}=$ benzothiazole) was isolated in $45 \%$ yield along with $30 \%$ of diamide 4 after column chromatographic purification. The isolation of $\mathbf{4}$ suggested that the reaction may initially proceeds via this intermediate, followed by ring closure to form the title compound. ${ }^{17}$ Furthermore, the yield of the desired product could be improved if complete conversion of diamide 4 to quinazolinone is possible. There are many instances which report the cyclization of diamides to quinazolinones under basic conditions. ${ }^{22}$ In some cases, pyridine has also been used for condensation of benzoxazinones with amines ${ }^{23}$ or for dehydrative cyclization of diamides to corresponding quinazolinones. ${ }^{24}$ However, the reaction proceeded sluggishly when pyridine alone was used as a solvent. This may be attributed to the less dielectric constant of pyridine. Therefore, we decided to use mixture of DMF and pyridine as solvent. In preliminary experiments with many different solvent ratios and power level, we observed that the most favorable one was DMF:Py (2:1) at $350 \mathrm{~W}$ (Scheme 2), which not only afforded the product in good yield (78\%), but also with better reaction rate (Table 2, Entry 4). Interestingly, the result was slightly better than solid support. This unexpected result encouraged us to extend this comparison for the synthesis of various (E)-3-benzothiazole/isoxazole substituted 2styrylquinazolin-4(3H)-one derivatives (1a-1u). The reaction time and yields of various quinazolinone derivatives for both the methods are compared in Table 3. 


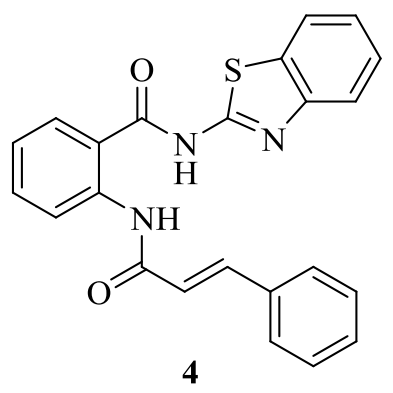

Table 2. Optimization of reaction conditions for the solution phase synthesis of (E)-3-(1,3benzothiazol-2-yl)-2-styrylquinazolin-4(3H)-one (1a) under microwave irradiation

\begin{tabular}{ccccc}
\hline Entry & $\begin{array}{c}\text { Solvent ratio } \\
\text { DMF:Py }\end{array}$ & $\begin{array}{c}\text { Power } \\
(\mathrm{W})\end{array}$ & $\begin{array}{c}\text { Reaction time } \\
(\min )\end{array}$ & $\begin{array}{c}\text { Yield }^{\text {a }} \\
(\%)\end{array}$ \\
\hline 1 & $1: 0$ & 350 & 7 & 45 \\
2 & $1: 2$ & 350 & 13 & 71 \\
3 & $1: 1$ & 350 & 12 & 75 \\
4 & $2: 1$ & 350 & 9 & 78 \\
5 & $2: 1$ & 280 & 15 & 78 \\
6 & $2: 1$ & 420 & 6 & 75 \\
\hline
\end{tabular}

${ }^{a}$ Isolated yield after recrystallization except entry 1 where column chromatography was used.

The results suggested that microwave assisted synthesis in presence of solvent was better than the solid phase reaction. The lesser yields in the solid supported method may be attributed to the decomposition of the benzoxazinones during the reaction, which was confirmed by the presence of corresponding $N$-acylanthranilic acid on the TLC plate.

All of the synthesized compounds were characterized by their physical, analytical and spectral data. They were given separately in experimental section. The MS (ESI) and NMR spectral data of all the synthesized compounds were in conformity with the structure assigned. Further, in the MS-ESI (positive mode) spectra exhibited molecular ion peak $\left([\mathrm{M}+\mathrm{H}]^{+}\right)$, appeared at different intensities, confirmed the exact mass or molecular weights of the examined compounds (1a-1l and 1m-au), while appearance of a characteristic two isotope peak $\left([(\mathrm{M}+\mathrm{H})+2]^{+}\right)$along with molecular ion peak $\left([\mathrm{M}+\mathrm{H}]^{+}\right)$in an intense ratio (almost 3:1 or 1:1) to the molecular ion peak confirmed the presence of halogen atoms of high abundance nature $(\mathrm{Cl}$ or $\mathrm{Br})$ in chloro-derivatives $(\mathbf{1 g}, \mathbf{1 r})$ or bromo-derivatives $(\mathbf{1} \mathbf{j}, \mathbf{1} \mathbf{u})$, respectively. 
Table 3. Synthesis of (E)-3-benzothiazole/isoxazole substituted-2-styrylquinazolin-4(3H)-one derivatives (1a-1u) under microwave irradiation

\begin{tabular}{|c|c|c|c|c|c|c|c|}
\hline \multirow{2}{*}{$\begin{array}{c}\text { Benzoxazin- } \\
\text { 4-ones } \\
(\mathbf{2 a - 2 i})^{\mathrm{a}} \\
\end{array}$} & \multirow{2}{*}{$\begin{array}{l}\text { Entitled } \\
\text { Compd. } \\
(\mathbf{1 a - 1 u})^{\text {a }}\end{array}$} & \multirow[b]{2}{*}{$\mathrm{Ar}$} & \multirow[b]{2}{*}{$\mathrm{Het}^{\mathrm{b}}$} & \multicolumn{2}{|c|}{ Acidic alumina ${ }^{c}$} & \multicolumn{2}{|c|}{$\mathrm{DMF} / \mathrm{Py}{ }^{\mathrm{d}}$} \\
\hline & & & & $\begin{array}{l}\text { Reaction } \\
\text { time (min) }\end{array}$ & $\begin{array}{c}\text { Yield }^{\mathrm{e}} \\
(\%)\end{array}$ & $\begin{array}{c}\text { Reaction } \\
\text { time (min) }\end{array}$ & $\begin{array}{c}\text { Yield }^{\mathrm{e}} \\
(\%)\end{array}$ \\
\hline $2 \mathrm{a}$ & 1a & $\mathrm{C}_{6} \mathrm{H}_{5}$ & $\mathrm{Bt}$ & 7 & 67 & 9 & 78 \\
\hline $2 \mathrm{a}$ & $1 \mathrm{~b}$ & $\mathrm{C}_{6} \mathrm{H}_{5}$ & 6-OMeBt & 6 & 62 & 8 & 81 \\
\hline $2 b$ & $1 \mathrm{c}$ & 4-OMe- $\mathrm{C}_{6} \mathrm{H}_{4}$ & 6-OMeBt & 6 & 60 & 7 & 77 \\
\hline $2 \mathrm{c}$ & 1d & $2-\mathrm{NO}_{2}-\mathrm{C}_{6} \mathrm{H}_{4}$ & 6-OMeBt & 5 & 64 & 6 & 86 \\
\hline $2 d$ & 1e & $3-\mathrm{NO}_{2}-\mathrm{C}_{6} \mathrm{H}_{4}$ & 6-OMeBt & 5 & 65 & 5 & 88 \\
\hline $2 \mathrm{e}$ & 1f & $4-\mathrm{NO}_{2}-\mathrm{C}_{6} \mathrm{H}_{4}$ & 6-OMeBt & 6 & 63 & 6 & 85 \\
\hline $2 f$ & $1 g$ & $4-\mathrm{Cl}-\mathrm{C}_{6} \mathrm{H}_{4}$ & 6-OMeBt & 5 & 61 & 7 & 76 \\
\hline $2 \mathrm{~g}$ & $1 \mathrm{~h}$ & 4-Me- $\mathrm{C}_{6} \mathrm{H}_{4}$ & 6-OMeBt & 7 & 65 & 8 & 75 \\
\hline $2 \mathrm{~h}$ & 1i & 2,3,4-tri-OMe- $\mathrm{C}_{6} \mathrm{H}_{3}$ & 6-OMeBt & 4 & 55 & 6 & 67 \\
\hline $2 \mathrm{i}$ & $\mathbf{1 j}$ & $2-\mathrm{NO}_{2}-\mathrm{C}_{6} \mathrm{H}_{4}$ & 6-OMeBt & 5 & 56 & 5 & 92 \\
\hline $2 \mathrm{c}$ & $1 \mathrm{k}$ & $2-\mathrm{NO}_{2}-\mathrm{C}_{6} \mathrm{H}_{4}$ & $\mathrm{Bt}$ & 6 & 68 & 7 & 84 \\
\hline $2 \mathrm{~g}$ & 11 & $4-\mathrm{Me}-\mathrm{C}_{6} \mathrm{H}_{4}$ & $6-\mathrm{NO}_{2} \mathrm{Bt}$ & 8 & 59 & 9 & 72 \\
\hline $2 \mathrm{a}$ & $1 \mathbf{m}$ & $\mathrm{C}_{6} \mathrm{H}_{5}$ & Ox & 5 & 65 & 7 & 80 \\
\hline $2 b$ & 1n & 4-OMe- $\mathrm{C}_{6} \mathrm{H}_{4}$ & Ox & 6 & 63 & 8 & 76 \\
\hline $2 \mathrm{c}$ & 10 & $2-\mathrm{NO}_{2}-\mathrm{C}_{6} \mathrm{H}_{4}$ & Ox & 7 & 67 & 6 & 84 \\
\hline $2 d$ & $1 p$ & $3-\mathrm{NO}_{2}-\mathrm{C}_{6} \mathrm{H}_{4}$ & Ox & 8 & 66 & 7 & 85 \\
\hline $2 \mathrm{e}$ & $1 q$ & $4-\mathrm{NO}_{2}-\mathrm{C}_{6} \mathrm{H}_{4}$ & Ox & 7 & 68 & 6 & 81 \\
\hline $2 \mathrm{f}$ & $1 \mathrm{r}$ & 4-Cl- $\mathrm{C}_{6} \mathrm{H}_{4}$ & Ox & 9 & 62 & 9 & 74 \\
\hline $2 \mathrm{~g}$ & $1 \mathrm{~s}$ & 4-Me- $\mathrm{C}_{6} \mathrm{H}_{4}$ & Ox & 5 & 61 & 8 & 72 \\
\hline $2 \mathrm{~h}$ & $1 t$ & 2,3,4-tri-OMe- $\mathrm{C}_{6} \mathrm{H}_{3}$ & Ox & 8 & 57 & 9 & 65 \\
\hline $2 \mathrm{i}$ & 1u & $2-\mathrm{NO}_{2}-\mathrm{C}_{6} \mathrm{H}_{4}$ & Ox & 9 & 62 & 6 & 84 \\
\hline
\end{tabular}

${ }^{a}$ Structure $\mathbf{1}$ and $\mathbf{2}$ in Scheme 2, where $\mathrm{X}=\mathrm{H}$ for all the compounds (except compounds $\mathbf{j}$ and $\mathbf{u}$, where $\mathrm{X}=\mathrm{Br}$ ). ${ }^{\mathrm{b}} \mathrm{Bt}=$ Benzothiazole and $\mathrm{Ox}=5$-tert-butylisoxazole. ${ }^{\mathrm{c}} 4 \mathrm{~g}$ of acidic alumina for 0.02 mol starting material, MWI at $455 \mathrm{~W} .{ }^{\mathrm{d}}$ In presence of DMF : Pyridine (2 : 1), MWI at 350 W. ${ }^{\mathrm{e}}$ Isolated yield after recrystallization.

\section{Experimental Section}

Column chromatographic separations were carried out on silica gel (60-120 mesh). The elemental analyses $(\mathrm{C}, \mathrm{H}$ and $\mathrm{N})$ were performed with a model Elemental Vario EL analyzer. The ${ }^{1} \mathrm{H}$ NMR and ${ }^{13} \mathrm{C}$ NMR spectra were recorded on a Bruker $(400 \mathrm{MHz})$ instrument using DMSO- $d_{6}$ as a solvent as well as an internal reference standard. The mass spectra (ESI-positive mode) were recorded on a Shimadzu LC-MS 2010 eV mass spectrophotometer in acetonitrile. 
Melting points are uncorrected. All the microwave assisted reactions were carried out at atmospheric pressure using a multimode microwave reactor (Microwave Synthesis System, Model: Cata-R, CatalystTM Systems, Pune-India) with attachment of glass vessel prolonged by a reflux condenser with constant stirring, whereby microwaves are generated by magnetron at a frequency of $2450 \mathrm{MHz}$ having an output energy range of 140 to 700 Watts, and the temperature was monitored with an external flexible probe. Acidic alumina (150 mesh; $58^{\circ} \mathrm{A}$; surface area 55 $\mathrm{m}^{2} / \mathrm{g}$; $\mathrm{pH}$ of aqueous suspension is $5.5 \pm 0.5$ ), 2-amino-1,3-benzothiazole, 2-amino-6-nitro-1,3benzothiazole and 3-amino-5-tert-butylisoxazole were purchased from Sigma-Aldrich Inc. Mumbai.

\section{Synthesis of 2-amino-6-methoxy-1,3-benzothiazole}

It was prepared by following a reported method. ${ }^{25}$ Accordingly, a mixture of $p$-anisidine (12.32 $\mathrm{g}, 100 \mathrm{mmol})$ and potassium thiocyanate $(14.58 \mathrm{~g}, 150 \mathrm{mmol})$ in glacial acetic acid (50 ml) was cooled to $5{ }^{\circ} \mathrm{C}$ and stirred vigorously. To this solution, bromine $(5.1 \mathrm{ml}, 100 \mathrm{mmol})$ in glacial acetic acid $(15 \mathrm{ml})$ was added drop wise at such a rate that the temperature did not increase more than $10{ }^{\circ} \mathrm{C}$ throughout the addition. Stirring was continued for an additional period of $2 \mathrm{~h}$ and the separated hydrobromide salt was filtered, dried and washed with acetic acid. It was subsequently dissolved in hot water and neutralized with an aqueous solution of ammonia. A solid thus separated out was filtered, washed with water and recrystallized from diluted ethanol to give the desired product in $75 \%$ yield. The product melted in the range of $165-167{ }^{\circ} \mathrm{C}$.

\section{General procedure for the synthesis of $(E)$-2-styryl-substituted-4H-[3,1]-benzoxazin-4- one derivatives}

The benzoxazinone derivatives were prepared by following the reported method. ${ }^{16}$ Appropriate anthranilic acid derivative $(10 \mathrm{mmol})$ contained in a round bottomed flask was dissolved in freshly dried pyridine $(5 \mathrm{ml})$. To the above solution, appropriate cinnamoyl chloride $(20 \mathrm{mmol})$ was added drop wise under stirring, over a period of $15 \mathrm{~min}$. The reaction mass was then allowed to stand for additional 2-3 h, with occasional shaking. It was then poured into $100 \mathrm{ml}$ ice cold water. The solid thus obtained was filtered, washed with cold water and recrystallized from ethanol.

(E)-2-styryl-4H-[3,1]-benzoxazin-4-one (2a) mp 144-146 ${ }^{\circ} \mathrm{C}$ (lit. ${ }^{16 \mathrm{~d}} \mathrm{mp}$ 147-148 $\left.{ }^{\circ} \mathrm{C}\right)$. IR ( $\mathrm{KBr}$, $\left.\mathrm{cm}^{-1}\right): 1762,1676,1642,1612$.

(E)-2-(4-methoxystyryl)-4H-[3,1]-benzoxazin-4-one (2b) mp $166-168{ }^{\circ} \mathrm{C}$ (lit. ${ }^{16 \mathrm{e}} \mathrm{mp} \quad 160^{\circ} \mathrm{C}$ ). IR $\left(\mathrm{KBr}, \mathrm{cm}^{-1}\right): 2814,1768,1670,1635,1605,1384$.

(E)-2-(2-nitrostyryl)-4H-[3,1]-benzoxazin-4-one (2c) mp 206-208 ${ }^{\circ} \mathrm{C}$ (lit. ${ }^{16 \mathrm{f}} \mathrm{mp} 206{ }^{\circ} \mathrm{C}$ ). IR $\left(\mathrm{KBr}, \mathrm{cm}^{-1}\right): 1765,1679,1638,1610,1545,1391$.

(E)-2-(3-nitrostyryl)-4H-[3,1]-benzoxazin-4-one (2d) mp 283-286 ${ }^{\circ} \mathrm{C}$ (lit. ${ }^{16 \mathrm{e}} \mathrm{mp} 283{ }^{\circ} \mathrm{C}$ ). IR $\left(\mathrm{KBr}, \mathrm{cm}^{-1}\right): 1764,1677,1641,1608,1548,1388$.

(E)-2-(4-nitrostyryl)-4H-[3,1]-benzoxazin-4-one (2e) mp $223-225{ }^{\circ} \mathrm{C}$ (lit. ${ }^{16 \mathrm{f}} \mathrm{mp} 221{ }^{\circ} \mathrm{C}$ ). IR $\left(\mathrm{KBr}, \mathrm{cm}^{-1}\right):$ 1768, 1680, 1637, 1604, 1547, 1392. 
(E)-2-(4-chlorostyryl)-4H-[3,1]-benzoxazin-4-one (2f) mp 200-203 ${ }^{\circ} \mathrm{C}$ (lit. ${ }^{16 \mathrm{e}} \mathrm{mp} 202{ }^{\circ} \mathrm{C}$ ). IR $\left(\mathrm{KBr}, \mathrm{cm}^{-1}\right): 1770,1679,1644,1610,714$.

(E)-2-(4-methylstyryl)-4H-[3,1]-benzoxazin-4-one (2g) mp 158-160 ${ }^{\circ} \mathrm{C}$ (lit. ${ }^{16 \mathrm{e}} \mathrm{mp} 190{ }^{\circ} \mathrm{C}$ ). IR $\left(\mathrm{KBr}, \mathrm{cm}^{-1}\right): 1763,1684,1638,1609,1374$.

(E)-2-(3,4,5-trimethoxystyryl)-4H-[3,1]-benzoxazin-4-one (2h) mp 203-206 ${ }^{\circ} \mathrm{C}$. IR ( $\mathrm{KBr}$, $\left.\mathrm{cm}^{-1}\right): 2811,1761,1679,1641,1602,1389$.

(E)-6-bromo-2-(2-nitrostyryl)-4H-[3,1]-benzoxazin-4-one (2i) mp 234-237 ${ }^{\circ} \mathrm{C}$ (lit. ${ }^{16 \mathrm{~g}} \mathrm{mp}$ Not found). IR (KBr, cm $\left.{ }^{-1}\right): 1772,1686,1645,1609,1540,1393,565$

General procedure for the synthesis of 3-benzothiazole/isoxazole substituted 2-styrylquinazolin-4(3H)-one derivatives on solid support

In a beaker, appropriate benzoxazinone $(2)(2 \mathrm{mmol})$ and heterocyclic primary amine (3) (0.28 g, $2 \mathrm{mmol})$ and acidic alumina (4 g) were taken in ethanol $(5 \mathrm{ml})$. The solvent was then evaporated on water bath under continuous stirring. The mixture was then heated under microwave irradiation at $420 \mathrm{~W}$ for an appropriate time (Table 3). The mixture was then cooled and extracted with ethyl acetate $(3 \times 20 \mathrm{ml})$ and washed with distilled water $(20 \mathrm{ml})$, dil. $\mathrm{HCl}$ $(2 \times 20 \mathrm{~mL})$, aq. $\mathrm{NaHCO}_{3}(2 \times 20 \mathrm{ml})$ and distilled water $(20 \mathrm{ml})$ sequentially by liquid-liquid extraction. The organic layer was dried and the resulting crude product was further purified by recrystallization from THF. Yields are given in Table 3.

General procedure for the solution phase synthesis of 3-benzothiazole/isoxazole substituted 2-styrylquinazolin-4(3H)-one derivatives

Appropriate benzoxazinone (2) (2 mmol) and heterocyclic primary amine (3) (0.28 g, $2 \mathrm{mmol})$ were taken in a solution of DMF $(1 \mathrm{ml})$ and pyridine $(0.5 \mathrm{ml})$ contained in a two-neck round bottomed flask fitted with a device condenser. The mixture was then heated under microwave irradiation at $350 \mathrm{~W}$ for an appropriate time (Table 3). After cooling, the reaction mass was dissolved in ethyl acetate $(40 \mathrm{ml})$ and washed with distilled water $(20 \mathrm{ml})$, dil. $\mathrm{HCl}(2 \times 20 \mathrm{~mL})$, aq. $\mathrm{NaHCO}_{3}(2 \times 20 \mathrm{ml})$ and distilled water $(20 \mathrm{ml})$ sequentially by liquid-liquid extraction. The organic layer was dried and the resulting crude product was further purified by recrystallization from THF. Yields are given in Table 3.

\section{Analyses}

(E)-3-(1,3-Benzothiazol-2-yl)-2-styrylquinazolin-4(3H)-one (1a). mp 221-223 ${ }^{\circ} \mathrm{C} . \mathrm{IR}(\mathrm{KBr}$, $\mathrm{cm}^{-1}$ ): $1687,1630,1606,689 .{ }^{1} \mathrm{H}$ NMR $\left(400 \mathrm{MHz}, \mathrm{DMSO}-d_{6}\right): \delta 6.91$ (d, J=15.6 Hz, 1H), 7.26$7.77(\mathrm{~m}, 11 \mathrm{H}), 7.90(\mathrm{bs}, 1 \mathrm{H}), 8.0(\mathrm{~d}, J=8.0 \mathrm{~Hz}, 1 \mathrm{H}), 8.07$ (bs, 1H). ${ }^{13} \mathrm{C}$ NMR (100 MHz, DMSO$\left.d_{6}\right): \delta 119.25,122.31,122.65,122.97,123.75,124.11,124.74,126.74,128.44,129.44,130.06$, 130.40, 132.78, 135.0, 136.72, 137.01, 141.27, 147.25, 148.73, 159.81, 164.11. MS (ESI) (m/z): $382.5[\mathrm{M}+\mathrm{H}]^{+}$. Anal. Calcd. for $\mathrm{C}_{23} \mathrm{H}_{15} \mathrm{~N}_{3} \mathrm{OS}$ : C, 72.42; H, 3.96; N, 11.02. Found: C, 72.35; H, $4.13 ; \mathrm{N}, 10.98$. 
(E)-3-(6-Methoxy-1,3-benzothiazol-2-yl)-2-styrylquinazolin-4(3H)-one (1b). mp 232-235 ${ }^{\circ} \mathrm{C}$. IR $\left(\mathrm{KBr}, \mathrm{cm}^{-1}\right): 2812,1698,1637,1596,1383,686 .{ }^{1} \mathrm{H}$ NMR (400 MHz, DMSO-d $)$ ): $\delta 3.92$ (s, $3 \mathrm{H}), 6.65(\mathrm{~d}, J=15.6 \mathrm{~Hz}, 1 \mathrm{H}), 6.76(\mathrm{~d}, J=8.8 \mathrm{~Hz}, 1 \mathrm{H}), 7.23-7.81(\mathrm{~m}, 11 \mathrm{H}), 8.03(\mathrm{~d}, J=8.0 \mathrm{~Hz}$, $1 \mathrm{H}) .{ }^{13} \mathrm{C}$ NMR $\left(100 \mathrm{MHz}, \mathrm{DMSO}-d_{6}\right): \delta 56.05,115.87,116.75,119.91,120.06,122.29,126.46$, 127.74, 127.89, 127.96, 128.31, 128.73, 132.68, 136.48, 138.96, 146.41, 147.57, 148.02, 160.34. MS (ESI) $(\mathrm{m} / z): 412.1[\mathrm{M}+\mathrm{H}]^{+}$. Anal. Calcd. for $\mathrm{C}_{24} \mathrm{H}_{17} \mathrm{~N}_{3} \mathrm{O}_{2} \mathrm{~S}: \mathrm{C}, 70.05 ; \mathrm{H}, 4.16 ; \mathrm{N}, 10.21$. Found: C, 70.15; H, 4.13; N, 10.18 .

(E)-3-(6-Methoxy-1,3-benzothiazol-2-yl)-2-(4-methoxystyryl)quinazolin-4(3H)-one (1c). mp 245-248 ${ }^{\circ} \mathrm{C}$. IR $\left(\mathrm{KBr}, \mathrm{cm}^{-1}\right): 2816,1704,1639,1609,1387,684 .{ }^{1} \mathrm{H}$ NMR (400 MHz, DMSO$\left.d_{6}\right): \delta 3.87(\mathrm{~s}, 3 \mathrm{H}), 3.91(\mathrm{~s}, 3 \mathrm{H}), 6.72(\mathrm{~d}, J=16 \mathrm{~Hz}, 1 \mathrm{H}), 6.73(\mathrm{~d}, J=8.8 \mathrm{~Hz}, 1 \mathrm{H}), 6.76(\mathrm{~d}, J=8.8$ $\mathrm{Hz}, 2 \mathrm{H}), 7.33-7.76(\mathrm{~m}, 8 \mathrm{H}), 7.99(\mathrm{~d}, J=7.6 \mathrm{~Hz}, 1 \mathrm{H}) .{ }^{13} \mathrm{C}$ NMR (100 MHz, DMSO- $\left.d_{6}\right): \delta 56.05$, $57.14,114.26,115.87,116.75,120.06,120.97,122.29$, 126.69, 127.74, 127.96, 126.67, 128.73, 136.48, 137.36, 146.41, 147.57, 148.02, 160.12, 160.34. MS (ESI) $(\mathrm{m} / z): 442.2[\mathrm{M}+\mathrm{H}]^{+}$. Anal. Calcd. for $\mathrm{C}_{25} \mathrm{H}_{19} \mathrm{~N}_{3} \mathrm{O}_{3} \mathrm{~S}: \mathrm{C}, 68.01 ; \mathrm{H}, 4.34 ; \mathrm{N}, 9.52$. Found: C, 68.20; H, 4.39; N, 9.48.

(E)-3-(6-Methoxy-1,3-benzothiazol-2-yl)-2-(2-nitrostyryl)quinazolin-4(3H)-one (1d). mp 267-269 ${ }^{\circ} \mathrm{C}$. IR $\left(\mathrm{KBr}, \mathrm{cm}^{-1}\right): 2814,1686,1632,1594,1541,1384,677 .{ }^{1} \mathrm{H}$ NMR (400 MHz, DMSO-d $\left.d_{6}\right): \delta 3.93(\mathrm{~s}, 3 \mathrm{H}), 6.77(\mathrm{~d}, J=8.8 \mathrm{~Hz}, 1 \mathrm{H}), 7.15(\mathrm{~d}, J=15.6,1 \mathrm{H}), 7.35-7.81(\mathrm{~m}, 9 \mathrm{H}), 7.92$ $(\mathrm{d}, J=7.6 \mathrm{~Hz}, 1 \mathrm{H}), 8.12(\mathrm{~d}, J=7.6 \mathrm{~Hz}, 1 \mathrm{H}) .{ }^{13} \mathrm{C}$ NMR $\left(100 \mathrm{MHz}, \mathrm{DMSO}-d_{6}\right): \delta 56.05,115.87$, $116.75,120.06,122.29,122.67,127.13,127.41,127.74,127.96,128.73,129.37,130.19,130.42$, 131.51, 136.48, 146.41, 147.57, 147.89, 148.02, 160.34. MS (ESI) $(\mathrm{m} / \mathrm{z}): 457.1[\mathrm{M}+\mathrm{H}]^{+}$. Anal. Calcd. for $\mathrm{C}_{24} \mathrm{H}_{16} \mathrm{~N}_{4} \mathrm{O}_{4} \mathrm{~S}: \mathrm{C}, 63.15 ; \mathrm{H}, 3.53 ; \mathrm{N}, 12.27$. Found: C, 63.28; H, 3.57; N, 12.21 .

(E)-3-(6-Methoxy-1,3-benzothiazol-2-yl)-2-(3-nitrostyryl)quinazolin-4(3H)-one (1e). mp 273-276 ${ }^{\circ} \mathrm{C}$. IR $\left(\mathrm{KBr}, \mathrm{cm}^{-1}\right): 2813,1688,1631,1593,1545,1388,679 .{ }^{1} \mathrm{H}$ NMR (400 MHz, DMSO-d $\left.)_{6}\right): \delta .94(\mathrm{~s}, 3 \mathrm{H}), 6.78(\mathrm{~d}, J=8.8 \mathrm{~Hz}, 1 \mathrm{H}), 7.2(\mathrm{~d}, J=16.4 \mathrm{~Hz}, 1 \mathrm{H}), 7.43-7.79(\mathrm{~m}, 7 \mathrm{H})$, 8.1-8.32 (m, 3H), $8.45(\mathrm{~s}, 1 \mathrm{H}) .{ }^{13} \mathrm{C}$ NMR $\left(100 \mathrm{MHz}, \mathrm{DMSO}-d_{6}\right): \delta 56.05,115.87,116.75$, $119.35,120.06,121.64,122.29,124.23,125.39,127.74,127.96,128.73,133.87,136.48,136.71$, 139.14, 146.41, 146.82, 147.57, 148.02, 160.34. MS (ESI) $(\mathrm{m} / \mathrm{z}): 457.2[\mathrm{M}+\mathrm{H}]^{+}$. Anal. Calcd. for $\mathrm{C}_{24} \mathrm{H}_{16} \mathrm{~N}_{4} \mathrm{O}_{4} \mathrm{~S}: \mathrm{C}, 63.15 ; \mathrm{H}, 3.53 ; \mathrm{N}, 12.27$. Found: C, 63.02; H, 3.56; N, 12.35.

(E)-3-(6-Methoxy-1,3-benzothiazol-2-yl)-2-(4-nitrostyryl)quinazolin-4(3H)-one (1f). mp 281$284{ }^{\circ} \mathrm{C}$. IR $\left(\mathrm{KBr}, \mathrm{cm}^{-1}\right): 2815,1685,1632,1594,1542,1394,676 .{ }^{1} \mathrm{H}$ NMR (400 MHz, DMSO$\left.d_{6}\right): \delta 3.93(\mathrm{~s}, 3 \mathrm{H}), 6.77(\mathrm{~d}, J=8.8 \mathrm{~Hz}, 1 \mathrm{H}), 7.12(\mathrm{~d}, J=15.6 \mathrm{~Hz}, 1 \mathrm{H}), 7.43-7.76(\mathrm{~m}, 5 \mathrm{H}), 7.86(\mathrm{~d}$, $J=8.8 \mathrm{~Hz}, 2 \mathrm{H}), 7.99-8.13(\mathrm{~m}, 2 \mathrm{H}), 8.24(\mathrm{~d}, J=8.8 \mathrm{~Hz}, 2 \mathrm{H}) .{ }^{13} \mathrm{C}$ NMR $\left(100 \mathrm{MHz}, \mathrm{DMSO}-d_{6}\right): \delta$ $56.05,115.87,116.75,120.06,121.37,122.29$, 123.07, 127.74, 127.96, 128.23, 128.73, 136.48, 137.78, 139.69, 146.41, 147.57, 148.02, 148.82, 160.34. MS (ESI) $(\mathrm{m} / z): 457.1[\mathrm{M}+\mathrm{H}]^{+}$. Anal. Calcd. for $\mathrm{C}_{24} \mathrm{H}_{16} \mathrm{~N}_{4} \mathrm{O}_{4} \mathrm{~S}$ : C, 63.15; H, 3.53; N, 12.27. Found: C, 63.33; H, 3.57; N, 12.28.

(E)-2-(4-Chlorostyryl)-3-(6-methoxy-1,3-benzothiazol-2-yl)quinazolin-4(3H)-one (1g). $\mathrm{mp}$ 286-288 ${ }^{\circ} \mathrm{C}$. IR (KBr, cm$\left.{ }^{-1}\right): 2814,1689,1633,1596,1383,712,681 .{ }^{1} \mathrm{H}$ NMR (400 MHz, DMSO-d $\left.)_{6}\right): \delta 3.89(\mathrm{~s}, 3 \mathrm{H}), 6.75(\mathrm{~d}, J=8.8 \mathrm{~Hz}, 1 \mathrm{H}), 6.89$ (d, $\left.J=16 \mathrm{~Hz}, 1 \mathrm{H}\right), 7.18$ (dt, $J=7.6,1.3$ $\mathrm{Hz}, 1 \mathrm{H}), 7.41-7.79(\mathrm{~m}, 9 \mathrm{H}), 8.32(\mathrm{dd}, J=7.6 \mathrm{~Hz}, 1 \mathrm{H}) .{ }^{13} \mathrm{C}$ NMR $\left(100 \mathrm{MHz}, \mathrm{DMSO}-d_{6}\right): \delta 56.05$, $115.87,121.24,116.75,120.06,122.29,127.33,127.74,127.96,128.73,129.86,132.07,134.74$, 
136.48, 137.44, 146.41, 147.57, 148.02, 160.34. MS (ESI) $(\mathrm{m} / \mathrm{z}): 445.8[\mathrm{M}+\mathrm{H}]^{+}, 447.8$ $[(\mathrm{M}+\mathrm{H})+2]^{+}$. Anal. Calcd. for $\mathrm{C}_{24} \mathrm{H}_{16} \mathrm{ClN}_{3} \mathrm{O}_{2} \mathrm{~S}$ : C, 64.64; H, 3.62; N, 9.42. Found: C, 64.59; H, $3.67 ; \mathrm{N}, 9.49$.

(E)-3-(6-Methoxy-1,3-benzothiazol-2-yl)-2-(4-methylstyryl)quinazolin-4(3H)-one (1h). mp 238-240 ${ }^{\circ} \mathrm{C}$. IR $\left(\mathrm{KBr}, \mathrm{cm}^{-1}\right): 2816,1701,1637,1603,1386,686 .{ }^{1} \mathrm{H}$ NMR (400 MHz, DMSO$\left.d_{6}\right): \delta 2.35(\mathrm{~s}, 3 \mathrm{H}), 3.9(\mathrm{~s}, 3 \mathrm{H}), 6.67(\mathrm{~d}, J=16 \mathrm{~Hz}, 1 \mathrm{H}), 7.19(\mathrm{t}, J=7.6,1 \mathrm{H}), 7.32-7.79(\mathrm{~m}, 10 \mathrm{H})$, $8.0(\mathrm{~d}, J=7.6 \mathrm{~Hz}, 1 \mathrm{H}) .{ }^{13} \mathrm{C}$ NMR $\left(100 \mathrm{MHz}, \mathrm{DMSO}-d_{6}\right): \delta 20.48,56.05,115.87,116.75,120.06$, 120.53, 122.29, 127.37, 127.68, 127.74, 127.96, 128.73, 132.83, 136.48, 136.87, 141.56, 146.41, 147.57, 148.02, 160.34. MS (ESI) $(\mathrm{m} / \mathrm{z}): 426.2[\mathrm{M}+\mathrm{H}]^{+}$. Anal. Calcd. for $\mathrm{C}_{25} \mathrm{H}_{19} \mathrm{~N}_{3} \mathrm{O}_{2} \mathrm{~S}: \mathrm{C}, 70.57$; H, 4.50; N, 9.88. Found: C, 70.45; H, 4.46; N, 9.95.

(E)-3-(6-Methoxy-1,3-benzothiazol-2-yl)-2-(3,4,5-trimethoxystyryl)-quinazolin-4(3H)-one (1i). mp 249-252 (d) ${ }^{\circ} \mathrm{C}$. IR $\left(\mathrm{KBr}, \mathrm{cm}^{-1}\right): 2818,1703,1639,1607,1387,688 .{ }^{1} \mathrm{H}$ NMR (400 MHz, DMSO-d $)_{6}$ : $\delta 3.87$ (s, 3H), 3.88 (s, 3H), 3.90 (s, 6H), 6.54 (d, J=16 Hz, 1H), 6.74 (d, J=8.8 $\mathrm{Hz}, 1 \mathrm{H}), 6.91(\mathrm{~s}, 2 \mathrm{H}), 7.42-7.82(\mathrm{~m}, 6 \mathrm{H}), 8.18(\mathrm{~d}, J=7.6 \mathrm{~Hz}, 1 \mathrm{H}) .{ }^{13} \mathrm{C}$ NMR $(100 \mathrm{MHz}, \mathrm{DMSO}-$ $\left.d_{6}\right): \delta 56.05,61.11,66.38,103.86,115.87,116.75,119.05,120.06,122.29,127.74,127.96$, 128.73, 130.12, 136.48, 146.39, 141.46, 141.65, 147.57, 148.02, 152.75, 160.34. MS (ESI) (m/z): $502.2[\mathrm{M}+\mathrm{H}]^{+}$. Anal. Calcd. for $\mathrm{C}_{27} \mathrm{H}_{23} \mathrm{~N}_{3} \mathrm{O}_{5} \mathrm{~S}: \mathrm{C}, 64.66 ; \mathrm{H}, 4.62 ; \mathrm{N}, 8.38$. Found: C, 64.52; H, $4.67 ; \mathrm{N}, 8.45$.

(E)-6-Bromo-3-(6-methoxy-1,3-benzothiazol-2-yl)-2-(2-nitrostyryl)-quinazolin-4(3H)-one (1j). mp 289-291 ${ }^{\circ} \mathrm{C}$. IR (KBr, cm $\left.{ }^{-1}\right): 2813,1684,1637,1594,1541,1392,689,564 .{ }^{1} \mathrm{H}$ NMR (400 MHz, DMSO-d $d_{6}$ : $\delta 3.94(\mathrm{~s}, 3 \mathrm{H}), 6.78$ (d, J=8.8 Hz, 1H), 7.18 (d, J=15.6 Hz, 1H), 7.43$7.97(\mathrm{~m}, 8 \mathrm{H}), 8.08-8.12(\mathrm{~m}, 2 \mathrm{H}) .{ }^{13} \mathrm{C}$ NMR (100 MHz, DMSO- $\left.d_{6}\right): \delta 56.05,115.87,116.75$, 119.29, 120.06, 122.68, 123.45, 123.91, 127.27, 127.41, 129.70, 130.24, 130.51, 131.26, 131.37, $139.74,147.35,147.89,147.94,148.02,159.14$. MS (ESI) $(m / z): 535.4[\mathrm{M}+\mathrm{H}]^{+}, 537.4$ $[(\mathrm{M}+\mathrm{H})+2]^{+}$. Anal. Calcd. for $\mathrm{C}_{24} \mathrm{H}_{15} \mathrm{BrN}_{4} \mathrm{O}_{4} \mathrm{~S}: \mathrm{C}, 53.84 ; \mathrm{H}, 2.82 ; \mathrm{N}, 10.47$. Found: C, 54.52; H, $2.67 ; \mathrm{N}, 10.95$.

(E)-3-(1,3-Benzothiazol-2-yl)-2-(2-nitrostyryl)quinazolin-4(3H)-one (1k). mp 243-246 ${ }^{\circ} \mathrm{C}$. IR $\left(\mathrm{KBr}, \mathrm{cm}^{-1}\right): 1682,1630,1606,1547,1385,682 .{ }^{1} \mathrm{H}$ NMR $\left(400 \mathrm{MHz}, \mathrm{DMSO}-d_{6}\right): \delta 7.04(\mathrm{t}$, $J=7.6 \mathrm{~Hz}, 1 \mathrm{H}), 7.19-7.99(\mathrm{~m}, 11 \mathrm{H}), 8.09-8.13(\mathrm{~m}, 2 \mathrm{H}) .{ }^{13} \mathrm{C}$ NMR $\left(100 \mathrm{MHz}, \mathrm{DMSO}-d_{6}\right): \delta$ $119.04,120.93,122.21,122.25,122.66,125.97,127.18,127.26,127.61,128.09,128.65,129.37$, $130.13,130.41,131.47,131.68,136.42,146.29,147.36,147.97,152.04,160.35,164.41 . \mathrm{MS}$ (ESI) $(m / z): 427.3[\mathrm{M}+\mathrm{H}]^{+}$. Anal. Calcd. for $\mathrm{C}_{23} \mathrm{H}_{14} \mathrm{~N}_{4} \mathrm{O}_{3} \mathrm{~S}$ : C, 64.78; H, 3.31; N, 13.14. Found: C, 63.56; H, 3.12; N, 13.97.

(E)-2-(4-Methylstyryl)-3-(6-nitro-1,3-benzothiazol-2-yl)quinazolin-4(3H)-one (11). mp 259$262{ }^{\circ} \mathrm{C}$. IR $\left(\mathrm{KBr}, \mathrm{cm}^{-1}\right): 1686,1633,1589,1541,1387,678 .{ }^{1} \mathrm{H}$ NMR (400 MHz, DMSO- $\left.d_{6}\right): \delta$ $2.35(\mathrm{~s}, 3 \mathrm{H}), 6.68(\mathrm{~d}, J=16 \mathrm{~Hz}, 1 \mathrm{H}), 7.33-7.78(\mathrm{~m}, 8 \mathrm{H}), 7.88-7.99(\mathrm{~m}, 3 \mathrm{H}), 8.89(\mathrm{~s}, 1 \mathrm{H}) .{ }^{13} \mathrm{C}$ NMR (100 MHz, DMSO- $\left.d_{6}\right): \delta 20.49,117.42,116.89,120.61,121.74,122.26,127.68,127.87$, $128.57,128.15,130.48,132.31,136.46,136.88,140.53,141.57,146.29,147.36,157.68,160.31$, 166.14. MS (ESI) $(\mathrm{m} / z): 441.1[\mathrm{M}+\mathrm{H}]^{+}$. Anal. Calcd. for $\mathrm{C}_{23} \mathrm{H}_{14} \mathrm{~N}_{4} \mathrm{O}_{3} \mathrm{~S}: \mathrm{C}, 65.44 ; \mathrm{H}, 3.66 ; \mathrm{N}$, 12.72. Found: C, 64.56; H, 3.72; N, 11.97 . 
(E)-3-(5-tert-Butylisoxazol-3-yl)-2-styrylquinazolin-4(3H)-one (1m). mp 152-154 ${ }^{\circ} \mathrm{C}$. IR $\left(\mathrm{KBr}, \mathrm{cm}^{-1}\right): 1683,1633,1591,1471,1449,1372 .{ }^{1} \mathrm{H}$ NMR (400 MHz, DMSO-d 6 ): $\delta 1.39$ (s, $9 \mathrm{H}), 6.65(\mathrm{~d}, J=15.6,1 \mathrm{H}), 7.19-7.24(\mathrm{~m}, 3 \mathrm{H}), 7.43-7.55(\mathrm{~m}, 3 \mathrm{H}), 7.68-7.73(\mathrm{~m}, 2 \mathrm{H}), 8.3(\mathrm{~d}$, $J=7.6 \mathrm{~Hz}, 1 \mathrm{H}) .{ }^{13} \mathrm{C}$ NMR $\left(100 \mathrm{MHz}\right.$, DMSO- $\left.d_{6}\right): \delta 29.26,32.18,90.63,119.83,122.28,126.69$, 127.58, 127.89, 128.12, 128.16, 128.47, 132, 83, 136.35, 138.89, 146.11, 147.15, 147.98, 160.34, 161.47. MS (ESI) $(m / z): 372.2[\mathrm{M}+\mathrm{H}]^{+}$. Anal. Calcd. for $\mathrm{C}_{23} \mathrm{H}_{21} \mathrm{~N}_{3} \mathrm{O}_{2}: \mathrm{C}, 74.37 ; \mathrm{H}, 5.70 ; \mathrm{N}$, 11.31. Found: C, 74.21; H, 5.66; N, 11.40 .

(E)-3-(5-tert-Butylisoxazol-3-yl)-2-(4-methoxystyryl)quinazolin-4(3H)-one (1n). mp 167-169 ${ }^{\circ} \mathrm{C}$. IR $\left(\mathrm{KBr}, \mathrm{cm}^{-1}\right): 2813,1698,1635,1597,1475,1454,1385,1373 .{ }^{1} \mathrm{H}$ NMR (400 MHz, DMSO- $\left.d_{6}\right): \delta 1.38(\mathrm{~s}, 9 \mathrm{H}), 3.87(\mathrm{~s}, 3 \mathrm{H}), 6.7-6.74(\mathrm{~m}, 3 \mathrm{H}), 7.21(\mathrm{~s}, 1 \mathrm{H}), 7.33-7.54(\mathrm{~m}, 4 \mathrm{H}), 7.68-$ $7.73(\mathrm{~m}, 2 \mathrm{H}), 8.27$ (d, $J=7.6 \mathrm{~Hz}, 1 \mathrm{H}) .{ }^{13} \mathrm{C}$ NMR (100 MHz, DMSO- $\left.d_{6}\right): \delta 29.26,32.18,57.06$, 90.63, 114.08, 120.81, 122.28, 126.53, 127.58, 128.12, 128.47, 128.79, 136.35, 137.67, 146.11, 147.15, 147.98, 160.18, 160.34, 161.47. MS (ESI) $(\mathrm{m} / \mathrm{z}): 402.2[\mathrm{M}+\mathrm{H}]^{+}$. Anal. Calcd. for $\mathrm{C}_{24} \mathrm{H}_{23} \mathrm{~N}_{3} \mathrm{O}_{3}$ : C, 71.80; H, 5.77; N, 10.47. Found: C, 71.71; H, 5.72; N, 10.52.

(E)-3-(5-tert-Butylisoxazol-3-yl)-2-(2-nitrostyryl)quinazolin-4(3H)-one (1o). mp 202-205 ${ }^{\circ} \mathrm{C}$. IR $\left(\mathrm{KBr}, \mathrm{cm}^{-1}\right): 1683,1633,1591,1519,1471,1449,1393,1369 .{ }^{1} \mathrm{H}$ NMR (400 MHz, DMSO$\left.d_{6}\right): \delta 1.40(\mathrm{~s}, 9 \mathrm{H}), 7.16(\mathrm{~d}, J=15.6,1 \mathrm{H}), 7.21(\mathrm{~s}, 1 \mathrm{H}), 7.32(\mathrm{t}, J=8 \mathrm{~Hz}, 1 \mathrm{H}), 7.5-7.56(\mathrm{~m}, 3 \mathrm{H})$, $7.66(\mathrm{~d}, J=15.6,1 \mathrm{H}), 7.87(\mathrm{t}, J=7.6,1 \mathrm{H}), 8.02-8.13(\mathrm{~m}, 3 \mathrm{H}) .{ }^{13} \mathrm{C}$ NMR $\left(100 \mathrm{MHz}, \mathrm{DMSO}-d_{6}\right)$ : $\delta$ 29.26, 32.18, 90.63, 122.28, 122.66, 127.13, 127.29, 127.58, 128.12, 128.47, 129.1, 130.16, 130.22, 131.64, 136.35, 146.11, 147.15, 147.89, 147.98, 160.34, 161.47. MS (ESI) $(\mathrm{m} / \mathrm{z}): 417.2$ $[\mathrm{M}+\mathrm{H}]^{+}$. Anal. Calcd. for $\mathrm{C}_{23} \mathrm{H}_{20} \mathrm{~N}_{4} \mathrm{O}_{4}$ : C, 66.34; H, 4.84; N, 13.45. Found: C, 66.45; H, 4.80; N, 13.39.

(E)-3-(5-tert-Butylisoxazol-3-yl)-2-(3-nitrostyryl)quinazolin-4(3H)-one (1p). mp 215-218 ${ }^{\circ} \mathrm{C}$. IR $\left(\mathrm{KBr}, \mathrm{cm}^{-1}\right): 1683,1633,1591,1545,1472,1449,1393,1371 .{ }^{1} \mathrm{H}$ NMR (400 MHz, DMSO$\left.d_{6}\right): \delta 1.41(\mathrm{~s}, 9 \mathrm{H}), 6.94(\mathrm{~d}, J=15.6,1 \mathrm{H}), 7.54-7.67(\mathrm{~m}, 4 \mathrm{H}), 7.84-7.92(\mathrm{~m}, 3 \mathrm{H}), 8.26(\mathrm{~d}, J=7.6$, 2H), $8.47(\mathrm{~s}, 1 \mathrm{H}) .{ }^{13} \mathrm{C}$ NMR $\left(100 \mathrm{MHz}, \mathrm{DMSO}-d_{6}\right): \delta 29.26,32.18,90.64,117.14,122.10$, 122.26, 122.28, 124.46, 127.24, 128.77, 130.11, 133.52, 136.45, 136.74, 138.90, 146.41, 148.91, 156.40, 158.94, 161.47. MS (ESI) $(\mathrm{m} / \mathrm{z})$ : $417.3[\mathrm{M}+\mathrm{H}]^{+}$. Anal. Calcd. for $\mathrm{C}_{23} \mathrm{H}_{20} \mathrm{~N}_{4} \mathrm{O}_{4}: \mathrm{C}, 66.34$; H, 4.84; N, 13.45. Found: C, 66.25; H, 4.87; N, 13.49 .

(E)-3-(5-tert-Butylisoxazol-3-yl)-2-(4-nitrostyryl)quinazolin-4(3H)-one (1q). mp 231-233 ${ }^{\circ} \mathrm{C}$. IR $\left(\mathrm{KBr}, \mathrm{cm}^{-1}\right): 1674,1633,1588,1545,1472,1447,1388,1369 .{ }^{1} \mathrm{H}$ NMR (400 MHz, DMSO$\left.d_{6}\right): \delta 1.40(\mathrm{~s}, 9 \mathrm{H}), 7.15(\mathrm{~d}, J=15.6,1 \mathrm{H}), 7.22(\mathrm{~s}, 1 \mathrm{H}), 7.54-7.69(\mathrm{~m}, 3 \mathrm{H}), 7.88(\mathrm{~d}, J=8.8 \mathrm{~Hz}$, $2 \mathrm{H}), 7.98-8.12(\mathrm{~m}, 2 \mathrm{H}), 8.32(\mathrm{~d}, J=8.8 \mathrm{~Hz}, 2 \mathrm{H}) .{ }^{13} \mathrm{C}$ NMR $\left(100 \mathrm{MHz}, \mathrm{DMSO}-d_{6}\right): \delta 29.26$, $32.18,90.63,121.29,122.28,123.73,127.58,128.12,128.28,128.47,136.35,137.86,139.98$, 146.11, 147.15, 148.43, 147.98, 160.34, 161.47. MS (ESI) $(\mathrm{m} / \mathrm{z}): 417.2[\mathrm{M}+\mathrm{H}]^{+}$. Anal. Calcd. for $\mathrm{C}_{23} \mathrm{H}_{20} \mathrm{~N}_{4} \mathrm{O}_{4}$ : C, 66.34; H, 4.84; N, 13.45. Found: C, 66.45; H, 4.92; N, 13.52 .

(E)-3-(5-tert-Butylisoxazol-3-yl)-2-(4-chlorostyryl)quinazolin-4(3H)-one (1r). mp 243-246 ${ }^{\circ} \mathrm{C}$. IR (KBr, $\left.\mathrm{cm}^{-1}\right): 1689,1632,1594,1474,1451,1372,712 .{ }^{1} \mathrm{H}$ NMR (400 MHz, DMSO- $\left.d_{6}\right): \delta$ 1.38 (s, 9H), 6.9 (d, J=16 Hz, 1H), 7.17 (dt, J=7.6, $1.3 \mathrm{~Hz}, 1 \mathrm{H}), 7.2(\mathrm{~s}, 1 \mathrm{H}), 7.5-7.58(\mathrm{~m}, 4 \mathrm{H})$, 7.73-7.77 (m, 2H), $8.31(\mathrm{dd}, J=7.6 \mathrm{~Hz}, 2 \mathrm{H}) .{ }^{13} \mathrm{C}$ NMR (100 MHz, DMSO- $\left.d_{6}\right): \delta 29.26,32.18$, 
90.63, 121.46, 122.28, 127.32, 127.58, 128.12, 128.47, 132.71, 134.48, 136.35, 137.63, 146.11, 147.15, 147.98, 160.34, 161.47. MS (ESI) $(\mathrm{m} / z): 405.9[\mathrm{M}+\mathrm{H}]^{+}, 407.9[(\mathrm{M}+\mathrm{H})+2]^{+}$. Anal. Calcd. for $\mathrm{C}_{23} \mathrm{H}_{20} \mathrm{ClN}_{3} \mathrm{O}_{2}$ : C, 68.06; H, 4.97; N, 10.35. Found: C, 67.98; H, 5.10; N, 10.27.

(E)-3-(5-tert-Butylisoxazol-3-yl)-2-(4-methylstyryl)quinazolin-4(3H)-one (1s). mp 155-157 ${ }^{\circ} \mathrm{C}$. IR $\left(\mathrm{KBr}, \mathrm{cm}^{-1}\right): 1686,1596,1634,1476,1452,1386,1371 .{ }^{1} \mathrm{H}$ NMR (400 MHz, DMSO- $\left.d_{6}\right)$ : $\delta 1.39(\mathrm{~s}, 9 \mathrm{H}), 2.35(\mathrm{~s}, 3 \mathrm{H}), 6.66(\mathrm{~d}, J=16 \mathrm{~Hz}, 1 \mathrm{H}), 7.18(\mathrm{t}, J=7.6 \mathrm{~Hz}, 1 \mathrm{H}), 7.21(\mathrm{~s}, 1 \mathrm{H}), 7.32(\mathrm{~d}$, $J=8 \mathrm{~Hz}, 2 \mathrm{H}), 7.61(\mathrm{dd}, J=7.6,1.3 \mathrm{~Hz}, 1 \mathrm{H}), 7.72-7.81(\mathrm{~m}, 4 \mathrm{H}), 8.03(\mathrm{~d}, J=7.6 \mathrm{~Hz}, 1 \mathrm{H}) .{ }^{13} \mathrm{C}$ NMR (100 MHz, DMSO- $\left.d_{6}\right): \delta$ 20.47, 29.26, 32.18, 90.63, 120.67, 122.28, 127.58, 127.74, 127.88, $128.12,128.47,132.36,136.35,136.86,141.59,146.11,147.15,147.98,160.34,161.47 . \mathrm{MS}$ (ESI) $(m / z): 386.3[\mathrm{M}+\mathrm{H}]^{+}$. Anal. Calcd. for $\mathrm{C}_{24} \mathrm{H}_{23} \mathrm{~N}_{3} \mathrm{O}_{2}: \mathrm{C}, 74.78 ; \mathrm{H}, 6.01 ; \mathrm{N}, 10.90$. Found: C, $74.65 ; \mathrm{H}, 5.95 ; \mathrm{N}, 11.01$.

(E)-3-(5-tert-Butylisoxazol-3-yl)-2-(3,4,5-trimethoxystyryl)quinazolin-4(3H)-one (1t). mp 210-213 ${ }^{\circ} \mathrm{C}$. IR $\left(\mathrm{KBr}, \mathrm{cm}^{-1}\right): 2815,1702,1639,1598,1479,1455,1387,1372 .{ }^{1} \mathrm{H}$ NMR (400 MHz, DMSO- $\left.d_{6}\right): \delta 1.37$ (s, 9H), 3.87 (s, 3H), 3.9 (s, 6H), $6.53(\mathrm{~d}, J=16 \mathrm{~Hz}, 1 \mathrm{H}), 6.92(\mathrm{~s}, 2 \mathrm{H})$, $7.01(\mathrm{t}, J=7.6 \mathrm{~Hz}, 1 \mathrm{H}), 7.18(\mathrm{~s}, 1 \mathrm{H}), 7.47$ (d, J=7.6 Hz, 1H), 7.58 (t, J=7.6 Hz, 1H), 7.79 (d, J=16 $\mathrm{Hz}, 1 \mathrm{H}), 8.15$ (d, $J=7.6 \mathrm{~Hz}, 1 \mathrm{H}) .{ }^{13} \mathrm{C}$ NMR (100 MHz, DMSO- $\left.d_{6}\right): \delta 29.26,32.18,61.12,66.26$, 90.63, 103.69, 119.51, 122.28, 127.58, 128.12, 128.47, 130.22, 136.35, 141.43, 141.58, 146.11, 147.15, 147.98, 152.55, 160.34, 161.47. MS (ESI) $(\mathrm{m} / \mathrm{z}): 462.4[\mathrm{M}+\mathrm{H}]^{+}$. Anal. Calcd. for $\mathrm{C}_{26} \mathrm{H}_{27} \mathrm{~N}_{3} \mathrm{O}_{5}$ : C, 67.66; H, 5.90; N, 9.10. Found: C, 67.76; H, 5.94; N, 8.99.

(E)-6-Bromo-3-(5-tert-butylisoxazol-3-yl)-2-(2-nitrostyryl)quinazolin-4(3H)-one (1u). mp 259-261 ${ }^{\circ} \mathrm{C}$. IR $\left(\mathrm{KBr}, \mathrm{cm}^{-1}\right): 1685,1632,1593,1542,1471,1447,1386,1370,568 .{ }^{1} \mathrm{H}$ NMR (400 MHz, DMSO-d $): \delta 1.40(\mathrm{~s}, 9 \mathrm{H}), 7.18(\mathrm{~d}, J=16 \mathrm{~Hz}, 1 \mathrm{H}), 7.23(\mathrm{~s}, 1 \mathrm{H}), 7.71-7.74(\mathrm{~m}, 2 \mathrm{H})$, $7.87(\mathrm{t}, \mathrm{J}=7.2 \mathrm{~Hz}, 1 \mathrm{H}), 7.9-8.14(\mathrm{~m}, 5 \mathrm{H}) .{ }^{13} \mathrm{C}$ NMR (100 MHz, DMSO- $\left.d_{6}\right): \delta 29.26,32.18,90.63$, 119.23, 122.67, 123.45, 123.94, 127.11, 127.26, 129.09, 130.11, 130.23, 131.78, 139.24, 147.21, 147.49, 147.98, 148.06, 159.44, 161.47. MS (ESI) $(\mathrm{m} / \mathrm{z}): 495.3[\mathrm{M}+\mathrm{H}]^{+}, 497.3[(\mathrm{M}+\mathrm{H})+2]^{+}$. Anal. Calcd. for $\mathrm{C}_{23} \mathrm{H}_{19} \mathrm{BrN}_{4} \mathrm{O}_{4}$ : C, 55.77; H, 3.87; N, 11.31. Found: C, 56.36; H, 3.94; N, 11.99 .

\section{Acknowledgements}

We acknowledge the University Grants Commission [UGC F. No. 34-301/2008 (SR)], New Delhi, for the financial support. We are thankful to the Principal and management of V. P. \& R. P. T. P. Science College, Vallabh Vidyanagar for providing necessary research facilities. We are also grateful to SICART (V. V. Nagar) for IR; Oxygen Healthcare Research Pvt. Ltd. (Ahmedabad) for Mass and CDRI (Lucknow) for NMR analyses.

\section{References}

1. Xie, W.; Jin, Y.; Wang, P. G. Chem. Tech. 1999, 29, 23. 
2. (a) Kappe, C. O.; Dallinger, D. Mol. Divers. 2009, 13, 71. (b) Kappe, C. O.; Stadler, A. Microwave in Organic and Medicinal Chemistry; Wiley-VCH: Weinheim, 2005. (c) Lidström, P.; Tierney, J. P. Microwave-Assisted Organic Synthesis; Blackwell: Oxford, 2004. (d) Loupy, A. Microwaves in Organic Synthesis; Wiley-VCH: Weinheim, 2002.

3. (a) Kappe, C. O.; Dallinger, D. Nature Rev. Drug. Discov. 2006, 5, 51. (b) Shipe, W. D.; Wolkenberg, S. E.; Lindsley, C. W. Drug Discov. Today Tech. 2005, 2, 155. (c) Leadbeater, N. E. Chem. Commun. 2005, 2881. (d) Kappe, C. O. Angew. Chem. Int. Ed. 2004, 43, 6250.

4. (a) Blackwell, H. E. Org. Biomol. Chem. 2003, 1, 1251. (b) Al-Obeidi, F.; Austin, R. E.; Okonya, J. F.; Bond, D. R. S. Mini-Rev. Med. Chem. 2003, 3, 449. (c) Lange, T.; Lindell, S. Comb. Chem. High Throughput Screening 2005, 8, 595.

5. (a) Alloum Ben, A.; Labiad, B.; Villemin, D. J. Chem. Soc., Chem. Commun. 1989, 386387. (b) Tanaka, K.; Toda, F. Chem. Rev. 2000, 100, 1025.

6. (a) Kamal, A.; Bharathi, V. E.; Ramaiah, J. M.; Dastagiri, D.; Reddy, S. J.; Viswanath, A.; Sultana, F.; Pushpavalli, S. N. C. V. L.; Pal-Bhadra, M.; Srivastava, H. K.; Sastry, N. G.; Juvekar, A.; Sen, S.; Zingde, S. Bioorg. Med. Chem. 2010, 18, 526. (b) Laddha, S. S.; Wadodkar, S. G.; Meghal, S. K. Med. Chem. Res. 2009, 18, 268. (c) Kamal, A.; Reddy, K. L.; Devaiah, V.; Shankaraiah, N.; Rao, M. V. Mini-Rev. Med. Chem. 2006, 6, 71. (d) Malawska, B. Curr. Top. Med. Chem. 2005, 5, 69. (e) Padala, S. R.; Padi, P. R.; Thipireddy, V. Heterocycles 2003, 60, 183. (f) Clercq, E. R. D. Biomed. Health Res. 2002, 55, 272. (g) Nawrocka, W.; Stasko, J. J. Boll. Chim. Farm. 2002, 141, 84. (h) Sinha, S.; Srivastava, M. Prog. Drug. Res. 1994, 43, 143. (i) Gupta, C. M.; Bhaduri, A. P.; Khanna, N. M. J. Sci. Ind. Res. 1971, 30, 101. (j) Amin, A. H.; Mehta, D. R.; Samarth, S. S. Prog. Drug Res. 1970, 14, 218.

7. (a) Jiang, J. B.; Hesson, D. P.; Dusak, B. A.; Dexter, D. L.; Kang, G. J.; Hamel, E. J. Med. Chem. 1990, 33, 1721. (b) Raffa, D.; Edler, M. C.; Daidone, G.; Maggio, B.; Merickech, M.; Plescia, S.; Schillaci, D.; Bai, R.; Hamel, E. Eur. J. Med. Chem. 2004, 39, 299.

8. Liu, J.-F.; Kaselj, M.; Isome, Y.; Ye, P.; Sargent, K.; Sprague, K.; Cherrak, D.; Wilson, C. J.; Si, Y.; Yohannes, D.; S.-C., N. J. Comb. Chem. 2006, 8, 7.

9. (a) Sgobba, M.; Rastelli, G. Chem. Med. Chem. 2009, 4, 1399. (b) Park, H.; Kim, Y.-J.; Hahn, J.-S. Bioorg. Med. Chem. Lett. 2007, 17, 6345.

10. (a) Bhandari, S. V.; Deshmane, B. J.; Dangare, S. C.; Gore, S. T.; Raparti, V. T.; Khachane, C. V.; Sarkate, A. P. Pharmacologyonline 2008, 2, 604. (b) Welch, W. M.; Ewing, F. E.; Huang, J.; Menniti, F. S.; Pagnozzi, M. J.; Kelly, K.; Seymour, P. A.; Guanowsky, V.; Guhan, S.; Guinn, M. R.; Critchett, D.; Lazzaro, J.; Ganong, A. H.; DeVries, K. M.; Staigers, T. L.; Chenard, B. L. Bioorg. Med. Chem. Lett. 2001, 11, 177.

11. (a) Jatav, V.; Mishra, P.; Kashaw, S.; Stables, J. P. Eur. J. Med. Chem. 2008, 43, 135. (b) Jatav, V.; Mishra, P.; Kashaw, S.; Stables, J. P. Eur. J. Med. Chem. 2008, 43, 1945.

12. (a) Gupta, V.; Kashaw, S. K.; Jatav, V.; Mishra, P. Med. Chem. Res. 2008, 17, 205. (b) Jatav, V.; Kashaw, S.; Mishra, P. Med. Chem. Res. 2008, 17, 169. 
13. (a) Jagani, C. L.; Sojitra, N. A.; Vanparia, S. F.; Patel, T. S.; Dixit, R. B.; Dixit, B. C. Arkivoc 2011, (ix), 221. (b) Jagani, C. L.; Sojitra, N. A.; Vanparia, S. F.; Patel, T. S.; Dixit, R. B.; Dixit, B. C. J. Saudi Chem. Soc. (Accepted Manuscript DOI: 10.1016/j.jscs.2011.02.001) (c) Dixit, R. B.; Vanparia, S. F.; Patel, T. S.; Jagani, C. L.; Doshi, H. V.; Dixit, B. C.; Appl. Organomet. Chem. 2010, 24(5), 408. (d) Vanparia, S. F.; Patel, T. S.; Sojitra, N. A.; Jagani, C. L.; Dixit, B. C.; Patel, P. S.; Dixit, R. B.; Acta Chem. Slov. 2010, 57(3), 660.

14. Connolly, D. J.; Cusack, D.; O'Sullivan, T. P.; Guiry, P. J. Tetrahedron 2005, 61, 10153.

15. (a) Wang, L.; Xia, J.; Qin, F.; Qian, C.; Sun, J. Synthesis 2003, 1241. (b) Salehi, P.; Dabiri, M.; Zolfigol, M. A.; Baghbanzadeh, M. Tetrahdron Lett. 2005, 46, 7051. (c) Dandia, A.; Singh, R.; Sarawgi, P. J. Fluorine Chem. 2005, 126, 307. (d) Liu, J.-F.; Lee, J.; Dalton, A. M.; Bi, G.; Baldino, C. M.; McElory, E.; Brown, M. Tetrahedron Lett. 2005, 46, 1241. (e) Rad-Moghadam, K.; Mohseni, M. J. Chem. Res. (S) 2003, 487.

16. (a) Fathalla, O. A. E.-F. M.; Kassem, E. M. M.; Ibrahem, N. M.; Kamel, M. M. Acta Pol. Pharm.-Drug Res. 2008, 65, 11. (b) Naito, T.; Ninomia, I. Heterocycles 1984, 22, 1705. (c) Bain, D. I.; Smalley, R. K. J. Chem. Soc. C. 1968, 1593. (d) Tiwari, S. S.; Pandey, V. K. J. Indian Chem. Soc. 1975, 52, 736. (e) Rose, U. J. Heterocycl. Chem. 1991, 28, 2005. (f) Messiha, N. N.; Abdel-Kader, A. M. M.; Nosseir, M. H. Indian J. Chem. 1973, 11B, 738. (g) Said, M. M.; El-Enany, M. M.; Ghoneim, K. M. Bull. Faculty Pharmacy (Cairo Uni.) 1980, $19,233$.

17. Zhou, Y.; Murphy, D. E.; Sun, Z.; Gregor, V. E. Tetrahedron Lett. 2004, 45, 8049.

18. Kidwai, M.; Ruby; Rastogi, S. Indian J. Chem., Sect. B 2004, 43B, 423.

19. Roopan, S. M.; Maiyalagan, T.; Khan, F. N. Can. J. Chem. 2008, 86, 1019.

20. (a) Salehi, P.; Zolfigol, M. A.; Shirini, F.; Baghbanzadeh, M. Curr. Org. Chem. 2006, 10, 2171. (b) Roy, A. D.; Jayalakshmi, K.; Dasgupta, S.; Roy, R.; Mukhopadhyay, B. Magn. Reson. Chem. 2008, 46, 1119. (c) Salehi, P.; Dabiri, M.; Zolfigol, M. A.; Baghbanzadeh, M. Tetrahedron Lett. 2005, 46, 7051.

21. Lidström, P.; Tierney, J.; Wathey, B.; Westman, J. Tetrahedron 2001, 57, 9225.

22. (a) Brunton, S. A.; Stibbard, J. H. A.; Rubin, L. L.; Kruse, L. I.; Guicherit, O. M.; Boyd, E. A.; Price, S. J. Med. Chem. 2008, 51, 1108. (b) Kshirsagar, U. A.; Mhaske, S. B.; Argade, N. P. Tetrahedron Lett. 2007, 48, 3243. (c) He, F.; Snider, B. B. J. Org. Chem. 1999, 64, 1397.

23. (a) Laddha, S. S.; Wadodkar, S. G.; Meghal, S. K. Arkivoc 2006, (xi), 1. (b) Al-Obaid, A. M.; Abdel-Hamide, S. G.; El-Kashef, H. A.; Abdel-Aziz, A. A.-M.; El-Azab, A. S.; AlKhamees, H. A.; El-Subbagh, H. I. Eur. J. Med. Chem. 2009, 44, 2379. (c) Madkour, H. M. F. Arkivoc 2004, (i), 36.

24. Kalusa, A.; Chessum, N.; Jones, K. Tetrahedron Lett. 2008, 49, 5840.

25. Trapani, G.; Franco, M.; Latrofa, A.; Carotti, A.; Gerichi, G.; Serra, M.; Biggio, G.; Liso G. Eur. J. Med. Chem. 1996, 31, 575. 